\title{
Predictive Neuroimaging Features and Growth Characteristics of Pediatric Brain Tumors Using Pre- Diagnostic Neuroimaging
}

Shannon Marie Green ( $\nabla$ shannongreen207@gmail.com )

University of California San Diego https://orcid.org/0000-0003-4205-0009

Victoria Vuong

UC San Diego: University of California San Diego

Paritosh Khanna

Rady Children's Hospital: Rady Children's Hospital San Diego

John Crawford

Rady Children's Hospital: Rady Children's Hospital San Diego

\section{Research Article}

Keywords: pediatric neuroradiology, pediatric brain tumor, tumor growth, brain neoplasm, tumor growth rate, apparent diffusion coefficient

Posted Date: February 3rd, 2022

DOI: https://doi.org/10.21203/rs.3.rs-1307426/v1

License: (9) This work is licensed under a Creative Commons Attribution 4.0 International License. Read Full License 


\section{Abstract \\ Purpose}

To evaluate for predictive neuroimaging features of pediatric brain tumor development and quantify tumor growth characteristics.

\section{Methods}

Retrospective review of 1098 consecutive pediatric patients at a single institution with newly diagnosed brain tumors from January 2009 to October 2021 was performed. Pre-diagnostic and diagnostic neuroimaging features (e.g., tumor size, apparent diffusion coefficient (ADC) values), clinical presentations, and neuropathology were recorded. High- and low-grade tumor sizes were fit to linear and exponential growth regression models.

\section{Results}

Fourteen of 1098 patients (1\%) had neuroimaging before diagnosis of a brain tumor (8 females, mean age 8.1 years, imaging interval 0.2-8.7 years). Tumor types included low-grade glioma $(n=4)$, embryonal tumors $(n=3)$, ependymoma $(n=3)$, and others $(n=4)$. Pre-diagnostic imaging of corresponding tumor growth sites were abnormal in four cases $(28 \%)$ and demonstrated higher ADC values in diagnostically high-grade tumors $(p=0.05)$. Growth regression analyses demonstrated $R^{2}$-values of 0.92 and 0.91 using a linear model and 0.64 and 0.89 using an exponential model for high- and low-grade tumors, respectively; estimated minimum velocity of diameter expansion was $2.4 \mathrm{~cm} /$ year for high-grade tumors and $0.4 \mathrm{~cm} /$ year for low-grade tumors. High-grade tumors demonstrated faster growth rate of diameter and solid tumor volume compared to low-grade tumors ( $p=0.02, p=0.03$, respectively).

\section{Conclusions}

This is the first study to utilize pre-diagnostic neuroimaging to demonstrate that linear and exponential growth rate models can be used to estimate pediatric brain tumor growth velocity and should be validated in a larger cohort.

\section{Introduction}

Central nervous system tumors are the most common type of solid tumor in the pediatric population and are the leading cause of cancer-related mortality in childhood [1]. Noninvasive characterization of brain tumors has rapidly evolved in the past five years as neuroimaging techniques have advanced. For example, various magnetic resonance imaging (MRI) techniques have been used to discriminate tumor type, including apparent diffusion coefficient (ADC) value [2, 3], mass spectroscopy [4], magnetic 
resonance fingerprinting [5], perfusion imaging, and dynamic susceptibility contrast [6, 7]. Neuroimaging also has been used to assess growth characteristics of common brain tumors in adults, including highgrade gliomas [8-12] and meningiomas [13-15]. However, growth patterns of pediatric brain tumors have not been formally studied. Furthermore, no study has characterized pediatric brain tumors based on neuroimaging features before diagnosis to date.

There are advantages of evaluating pre-diagnostic neuroimaging. First, suspicious features can be critically evaluated. Presumably, the same diagnostic features used to classify brain tumors can be assessed on pre-diagnostic imaging in the region of subsequent tumor growth, e.g., enhancement, perfusion, ADC values. With increasing use of advanced diagnostic imaging and evolving imaging techniques, more opportunities exist to identify tissue at risk for tumor development.

Secondly, tumor growth patterns can be evaluated on pre-diagnostic neuroimaging. Thus far, the most widely accepted adult tumor growth models include: (1) exponential growth (i.e., constant volume doubling time); (2) linear growth (i.e., constant radial growth velocity); and (3) Gompertzian growth (i.e., sigmoid function), which assumes progressively decreased volume doubling time due to diminishing tumor nutrients. Of the three models, the Gompertzian model is most supported, with previous studies demonstrating a growth plateau $[8,13]$. However, no consensus for which model best estimates tumor growth exists secondary to the complexity and multifactorial nature of tumor development. For example, tumors with the same histopathology may have different growth characteristics based on molecular biomarkers [12], size at diagnosis [8, 14], aggressiveness [13], patient age [13], tumor location [14], and tumor region measured [12]. Furthermore, few studies have assessed early tumor growth patterns because tumors clinically present typically when macroscopic, limiting knowledge of the exact start of tumor development. By evaluating imaging before the brain tumor diagnosis, tumor characteristics, including growth pattern, can be further elucidated.

This study aimed to evaluate pediatric neuroimaging both before and at the time of brain tumor diagnosis to identify possible predictive imaging features for tumor development and to evaluate pediatric brain tumor growth patterns.

\section{Methods}

A retrospective review of 1098 consecutive pediatric patients with a brain tumor diagnosed between January 2009 and October 2021 at Rady Children's Hospital-San Diego were reviewed. Inclusion criteria included patients $\leq 18$ years old with cross-sectional neuroimaging (e.g., CT, MRI) before and at the time of tumor diagnosis. Patients with known cancer syndromes were excluded. Institutional Review Board approval was obtained.

\section{Clinical Information}

Demographic information was obtained, including gender, race, age, indication at time of neuroimaging, modality (i.e., CT, MRI), clinical presentation, and time interval between imaging studies. Patient 
management course and clinical outcome were recorded, including surgical, chemotherapy, and/or radiation. If applicable, degree of resection was noted.

Histopathologic diagnosis, if available, and corresponding World Health Organization (WHO) classification were recorded [16]. High-grade tumors and low-grade tumors were differentiated based on WHO grade and/or presence of anaplastic imaging and were analyzed separately.

\section{Neuroimaging Features}

All images were viewed and evaluated using the IBM Watson Health Merge PACS ${ }^{\mathrm{TM}}$ software, v7.1.2.154108. Tumor neuroimaging characteristics were recorded, including location, enhancement, total tumor size, and solid component size. All brain MRIs performed following diagnosis and before treatment were reviewed, including four low-grade tumors initially managed by observation. Total tumor volume was estimated using a general ellipsoid formula $\left(V=4 / 3 \pi^{*} a{ }^{*}{ }^{*} c\right)$, where $V$ represents the volume and the $a, b$, and $c$ variables represent half the tumor diameter in transverse, anteroposterior, and craniocaudal dimensions, respectively. The observed growth rates of each tumor by diameter and solid tumor volume were calculated using difference in tumor size between pre-diagnostic and diagnostic imaging divided by the time interval between imaging studies.

Mean $A D C$ values were measured using ellipsoid regions of interest (ROI). ROls were drawn on the largest area of regions of solid and/or enhancing tumor on the diagnostic MRI and the corresponding region on pre-diagnostic imaging (Figure 1a). ADC values were not available for six pre-diagnostic imaging studies, due to lack of MRI imaging $(n=4)$ or due to intraventricular tumor location $(n=2)$. Diffusion restriction could not be evaluated in two diagnostic neuroimaging studies due to susceptibility artifact.

\section{Statistical Methods}

Parametric data were expressed as mean[range] and compared using the Student's t-test. Nonparametric data were expressed as mean[range] and compared using the Welch's t-test. F-test was used to determine variance. All tests were 2-sided and were determined to be significant if the $p$-value $\leq 0.05$. The atypical teratoid/rhabdoid tumor (ATRT) was separated from tumor growth analyses and used as a reference for high-grade tumor growth rate given macroscopic tumor was visualized on the pre-diagnostic study retrospectively. The case of osteoblastic osteosarcoma was also excluded from growth analyses given tumor origination outside the craniospinal axis.

To model tumor growth for high- and low-grade tumor groups, best linear and exponential models were fit to plots of tumor size versus time interval between pre-diagnostic and diagnostic imaging studies, using diameter and solid tumor volume for size, respectively. Goodness of fit $\left(\mathrm{R}^{2}\right)$ of each model was recorded. The $y$-intercept was set at zero for the linear growth model to represent lack of visible tumor on the prediagnostic imaging.

Statistical analysis was performed using Excel ${ }^{\circledR}$, v2110 (Build 14527.20276 Click-to-Run). 


\section{Results}

Fourteen total patients (1\%) met inclusion criteria (Table 1). Mean patient age at tumor diagnosis was 8.1[2.8-14.5] years. Tumor types included ATRT $(n=1)$, ependymoma $(n=3)$, medulloblastoma $(n=1)$, low-grade glioma $(n=2)$, pineoblastoma $(n=1)$, pineocytoma $(n=1)$, osteoblastic osteosarcoma $(n=1)$, CNS lymphoma $(n=1)$, ganglioglioma $(n=1)$, and juvenile xanthogranuloma $(J X G)(n=1)$. Seven tumors were classified as high-grade tumors (Cases 1-7) and seven were classified as low-grade tumors (Cases 8-14). Mean interval time between pre-diagnostic and diagnostic imaging studies for all cases was $3.0[0.2-8.7]$ years. The mean interval time between studies was not statistically significant for high-grade versus low-grade tumors (1.8[0.2-3.1] years vs. 4.5[1.4 - 8.7] years, respectively, $p=0.1$, Table 1$)$. Notably, three low-grade gliomas managed by observation demonstrated no interval growth in size during the observation period, up to 2.5 years.

For high-grade tumors, the most common clinical presentation prompting diagnostic neuroimaging was headache/vomiting ( 5 of 7 cases). For low-grade tumors, seizure was the most common clinical presentation (4 of 7 cases). Neurologic complaints (e.g., involuntary movements, nystagmus) comprised the majority of the remaining clinical presentations. Two patients were diagnosed with tumors for incidental reasons (i.e., trauma workup, autism workup) and both measured less than $1.5 \mathrm{~mL}$ in volume at diagnosis. Notably, a higher proportion of the diagnostic neuroimaging studies were obtained as emergent studies (79\%) compared to the pre-diagnostic neuroimaging (36\%).

Neuroimaging features of the tumors are described in Table 2. Ten tumors had no pre-diagnostic imaging. Pre-diagnostic MRI was not available in four cases (28\%). Of these cases, one demonstrated thickened, enhancing choroid and subsequently developed an ATRT (Figure 1b). The remaining three cases were pathologically diagnosed as low-grade and demonstrated nonspecific findings on the prediagnostic neuroimaging at the site of subsequent tumor growth, including T2/FLAIR signal abnormality (Figure 1c), encephalomalacia (Figure 1d), or nonspecific white matter attenuation (Figure 1e).

The linear and exponential regression models are represented in Figure $2 \mathrm{a}$ and $2 \mathrm{c}$, respectively. The linear model had an excellent goodness of fit for high- and low-grade tumors $\left(R^{2}=0.92\right.$ and $R^{2}=0.91$, respectively). The exponential model demonstrated a reasonable goodness of fit for high-grade tumors $\left(R^{2}=0.64\right)$ and excellent goodness of fit for low-grade tumors $\left(R^{2}=0.89\right)$. For the high-grade tumor reference, the velocity of diameter expansion (VDE) was $1.8 \mathrm{~cm} /$ year (Figure 2a) and the volume doubling time was approximately 500 days (Figure 2c). The high-grade tumors demonstrated an average VDE of $2.4 \mathrm{~cm} /$ year (Figure 2a), reflecting the minimum VDE for high-grade tumors, and estimated volume doubling time of 158 days (Figure 2c). The low-grade tumors demonstrated a minimum VDE of 0.4 $\mathrm{cm} /$ year (Figure 2a) and volume doubling time of 806 days (Figure 2c). Of note, a low-grade ependymoma with neuroimaging studies following diagnosis and before treatment showed a VDE of 0.4 $\mathrm{cm} /$ year during the imaging period. The average growth rate of the high-grade tumors based on diameter and solid volume were significantly higher than low-grade tumors ( $p=0.02$ and $p=0.03$, respectively) (Figure $2 b$ and $2 d$ ). 
Mean ADC values of the high- and low-grade tumors on pre-diagnostic and diagnostic imaging are provided in Figure 3. Mean ADC value was $1.264\left[0.813-1.635 \times 10^{-3}\right] \mathrm{mm}^{2} / \mathrm{s}$ for low-grade tumors and $0.661\left[0.421-0.851 \times 10^{-3}\right] \mathrm{mm}^{2} / \mathrm{s}$ for high-grade tumors. The ADC values of the high-grade tumors on diagnostic imaging were significantly lower than the corresponding region on pre-diagnostic imaging $(p=$ $0.05)$, but similar on pre-diagnostic and diagnostic imaging for low-grade tumors $(p=0.87)$. On prediagnostic imaging, the ADC values were similar between the high- and low-grade tumors $(p=0.22)$, but on diagnostic imaging, high-grade tumors had lower ADC values than low-grade tumors $(p=0.002)$.

\section{Discussion}

This study is the first to evaluate pre-diagnostic neuroimaging of pediatric brain tumors. The primary goal of this retrospective study of 14 high- and low-grade pediatric brain tumors was to identify possible predictive neuroimaging characteristics, including growth rate. Evaluation of high-grade and low-grade tumor growth demonstrated an excellent fit using the linear growth rate regression model, with $\mathrm{R}^{2}=0.91$ - 0.92 for both tumor subtypes. Moreover, pre-diagnostic imaging allowed specific evaluation of ADC values, which was not significantly different between pre-diagnostic and diagnostic imaging for lowgrade tumors and was higher in pre-diagnostic imaging compared to diagnostic imaging for high-grade tumors.

Currently, quantitative studies of pediatric brain tumor growth is lacking. Multiple factors contribute to the sparsity of data on this topic, including a relatively lower incidence of pediatric brain tumors compared to the adult population, increased awareness of radiation safety for the pediatric population, and special considerations for pediatric MRI. Particularly, high-grade tumor growth is difficult to characterize given they are often managed immediately following diagnosis, limiting long-term treatment-naïve observation. However, several studies have assessed the growth characteristics of common high-grade tumors in adults [8-12]. For example, Ellingson et al. found that median volumetric doubling time of preoperative, treatment-naive high-grade gliomas was 21.1 days [8]. In addition, Fan et al. demonstrated an estimated VDE of $7.0 \mathrm{~cm} /$ year for glioblastomas and $5.1 \mathrm{~cm} /$ year for all high-grade gliomas [12]. Stensjøen et al. reported large variations in glioblastoma growth rates, with a median velocity of radial expansion (VRE) of $3.0 \mathrm{~cm} /$ year. The authors also noted that one-third of tumors doubled in volume between the diagnostic and preoperative scans, while another one-third were unchanged or decreased in volume [10]. Wang et al. also reported large variations in glioblastoma growth rates in vivo but demonstrated a VRE of $3.0 \mathrm{~cm} /$ year [11].

In this study, the estimated VDE was $2.4 \mathrm{~cm} /$ year, lower than previously reported values in the adult population. Given the exact time of initial tumor development is unknown, this calculated VDE is likely lower than the true VDE, partially accounting for the relatively slower growth rates compared to previously reported data. Another possible explanation is that the true growth rate model instead mimics the Gompertzian growth curve. If so, previously reported values would have been calculated using tumor sizes after they had passed the inflection point on the Gompertzian growth curve, falsely elevating the reported growth rate values. Finally, it is possible that tumor growth rate may be inherently slower in the 
pediatric population - a theory supported by the high-grade tumor reference, which demonstrated a VDE of $1.8 \mathrm{~cm} /$ year.

Conversely, previously reported low-grade growth characteristics are limited. Contemporaneous studies focus predominantly on analysis of adult low-grade tumor growth, including meningiomas [13-15] and low-grade gliomas [17] and demonstrate a wide range of growth patterns and rates. For example, Nakasu et al. reported volume doubling times of meningiomas ranging between 111 days and 91,400 days. Additionally, the authors noted the fastest growth rates in atypical meningiomas, intermediate growth rates in benign, noncalcified meningiomas, and slowest growth rates in calcified meningiomas. In comparison, the estimated volume doubling time in our study was 908 days, which was comparable to the intermediate growth rates presented by Nakasu et al. [15]. Given the excellent fit of low-grade tumor group to the linear growth model, the estimated minimum VDE calculated in this study, $0.4 \mathrm{~cm} /$ year, likely better estimates the true growth rate for low-grade tumors, further supported by analysis of the low-grade ependymoma growth rate after diagnosis but before treatment, also $0.4 \mathrm{~cm} /$ year.

Though tumor growth rates have only been described in the adult population thus far, ADC values have been used to characterize brain tumors in both pediatric and adult populations. For example, Novak et al. focused on the classification of pediatric brain tumors using ADC values. The authors showed characteristic $A D C$ values for ependymomas were $1.126 \pm 0.155 \times 10^{-3} \mathrm{~mm}^{2} / \mathrm{s}$ and $0.870 \pm 0.154 \times 10^{-3}$ $\mathrm{mm}^{2} / \mathrm{s}$ for medulloblastomas [3]. Similarly, Abdulaziz et al. demonstrated ADC values ranging between $0.225-1.240 \times 10^{-3} \mathrm{~mm}^{2} / \mathrm{s}$ for ependymal tumors, $0.107-1.571 \times 10^{-3} \mathrm{~mm}^{2} / \mathrm{s}$ for embryonal tumors, $0.5220-0.7840 \times 10^{-3} \mathrm{~mm}^{2} / \mathrm{s}$ for other astrocytic tumors, and $0.1530-0.8160 \times 10^{-3} \mathrm{~mm}^{2} / \mathrm{s}$ for meningiomas [2]. Yet these prior studies have not used pre-diagnostic imaging to characterize brain tissue at the site of subsequent tumor growth. In this study, the high-grade tumors demonstrated ADC values compatible with previously reported values.

Notably, this study did not demonstrate a lower mean ADC value in the pre-diagnostic neuroimaging studies of the high-grade tumors compared to the low-grade tumors. However, there was a small sample size in each tumor subtype, limiting analysis and the ability to draw definite conclusions about the utility of pre-diagnostic ADC values in characterizing brain tumors. Future studies with a larger sample size may further validate or negate this point.

Pediatric patients with brain tumors rarely receive brain imaging before diagnosis, limiting full characterization of different tumor subtypes. Thus far, adult tumor subtypes have been characterized using growth rate models and $A D C$ values of neuroimaging already demonstrating macroscopic tumor. Prior studies do not evaluate ADC values of the corresponding brain tissue on pre-diagnostic imaging. This study is the first to demonstrate that both linear and exponential growth rate models can be used to estimate the growth rate of pediatric brain tumors. In addition, this study analyzed the use of ADC values to characterize high- and low-grade tumor subtypes in both pre-diagnostic and diagnostic imaging. Overall, this study demonstrates increased opportunity to identify tissue that is at risk for tumor 
development with the use of advanced diagnostic imaging and possibly allows for an increased chance of early, curative management.

\section{Limitations}

This study has several limitations. First, the pre-diagnostic and diagnostic neuroimaging was retrospectively analyzed and subject to the pitfalls of all retrospective analyses. Additionally, despite demonstrating an absence of macroscopic tumor on pre-diagnostic neuroimaging, the precise timing of initial microscopic tumor appearance cannot be determined, limiting the determination of the exact rate of tumor growth.

The small sample size also results in several limitations. First, statistical evaluation of growth characteristics for each tumor type was limited. In addition, comparison of the three most common growth models could not be performed given the small sample size, limiting the ability to draw firm conclusions about the fit to a specific growth rate model. Particularly, the Gompertzian growth model could not be tested because only two studies (pre-diagnostic and diagnostic) were consistently obtained and there was an absence of macroscopic tumor on the pre-diagnostic study in most cases. Future studies with larger samples sizes of combined cohorts could further validate the accuracy of the fit to a particular growth model.

Moreover, although the imaging at time of diagnosis was uniform in terms of high quality and technique, consistent uniform modality, quality, and technique of pre-diagnostic images could not be achieved because some neuroimaging studies were performed at outside facilities. Notably, about a quarter of the pre-diagnostic imaging studies did not include MRI, which is more sensitive for detection of small tumors. If available, MRI could have provided more quantitative information on tumor growth.

Lastly, tumor segmentation software was not available at the study site. Therefore, tumor volumes were estimated using the ellipsoid model formula, and tumor ADC values were estimated using a best-fit ellipsoid ROI function tool within the imaging system. This prevented more exact evaluation of the tumors. Future studies with tumor software segmentation may be used to better estimate tumor volumes and $A D C$ values.

\section{Conclusion}

Evaluation of pre-diagnostic neuroimaging at sites of subsequent pediatric brain tumor growth demonstrated distinct growth characteristics in high-versus low-grade tumor subtypes using both linear and exponential growth rate models. The linear growth regression model had an excellent fit for high- and low-grade tumor growth compared to the reasonable fit with the exponential growth model, suggesting a greater correlation of using the velocity of diameter expansion to calculate the true growth rate rather than volume doubling time. Additionally, characterization of the tumor subsets on pre-diagnostic imaging demonstrated similar ADC values of high-grade tumors compared to low-grade tumors. Further studies 
with larger cohorts will need to be performed to compare growth rate models and determine which model best represents the true tumor growth rate, and to further examine the use of pre-diagnostic ADC values to fully characterize different tumor subtypes in the pediatric population.

\section{Abbreviations}

ADC = apparent diffusion coefficient; AMS = altered mental status; ATRT = atypical teratoid rhabdoid tumor; $\mathrm{CE}=$ contrast enhanced; chemo = chemotherapy; $\mathrm{CNS}=$ central nervous system; $\mathrm{DLBCL}=$ diffuse large $B$ cell lymphoma; $D X$ = diagnosis; $E D$ = emergency department; $E T V$ = endoscopic third ventriculostomy; GTR = gross total resection; $\mathrm{HA}$ = headache; JXG = juvenile xanthogranuloma; $\mathrm{LGG}=$ low-grade glioma; MRI = magnetic resonance imaging; NTR = near total resection; OSF = outside facility; $\mathrm{RT}=$ radiation therapy; STR = subtotal resection; VDE = velocity of diameter expansion

\section{Declarations}

All the authors of this article have reported no disclosures nor conflicts of interest. This research received no specific grant from any funding agency in the public, commercial, or not-for-profit sectors.

\section{References}

1. Udaka YT, Packer RJ (2018) Pediatric Brain Tumors. Neurol Clin 36:533-556 doi: S07338619(18)31206-4 [pii]

2. Al-Sharydah AM, Al-Arfaj HK, Saleh Al-Muhaish H, Al-Suhaibani SS, Al-Aftan MS, Almedallah DK, AlAbdulwahhab AH, Al-Hedaithy AA, Al-Jubran SA (2019) Can apparent diffusion coefficient values help distinguish between different types of pediatric brain tumors? Eur J Radiol Open 6:49-55. doi: 10.1016/j.ejro.2018.12.004[doi]

3. Novak J, Zarinabad N, Rose H, Arvanitis T, MacPherson L, Pinkey B, Oates A, Hales P, Grundy R, Auer D et al (2021) Classification of paediatric brain tumours by diffusion weighted imaging and machine learning. Sci Rep 11:2987-2021. doi: 10.1038/s41598-021-82214-3[doi]

4. Clark AR, Calligaris D, Regan MS, Pomeranz Krummel D, Agar JN, Kallay L, MacDonald T, Schniederjan M, Santagata S, Pomeroy SL et al (2018) Rapid discrimination of pediatric brain tumors by mass spectrometry imaging. J Neurooncol 140:269-279. doi: 10.1007/s11060-018-29782[doi]

5. de Blank P, Badve C, Gold DR, Stearns D, Sunshine J, Dastmalchian S, Tomei K, Sloan AE, BarnholtzSloan JS, Lane A et al (2019) Magnetic Resonance Fingerprinting to Characterize Childhood and Young Adult Brain Tumors. Pediatr Neurosurg 54:310-318. doi: 10.1159/000501696[doi]

6. Dallery F, Bouzerar R, Michel D, Attencourt C, Promelle V, Peltier J, Constans JM, Baledent O, GondryJouet C (2017) Perfusion magnetic resonance imaging in pediatric brain tumors. Neuroradiology 59:1143-1153. doi: 10.1007/s00234-017-1917-9[doi] 
7. Morana G, Tortora D, Stagliano S, Nozza P, Mascelli S, Severino M, Piatelli G, Consales A, Lequin M, Garre ML et al (2018) Pediatric astrocytic tumor grading: comparison between arterial spin labeling and dynamic susceptibility contrast MRI perfusion. Neuroradiology 60:437-446. doi: 10.1007/s00234-018-1992-6[doi]

8. Ellingson BM, Nguyen HN, Lai A, Nechifor RE, Zaw O, Pope WB, Yong WH, Nghiemphu PL, Liau LM, Cloughesy TF (2016) Contrast-enhancing tumor growth dynamics of preoperative, treatment-naive human glioblastoma. Cancer 122:1718-1727. doi: 10.1002/cncr.29957[doi]

9. Badve C, Sloan AE (2015) Modeling the growth dynamics of glioblastoma using magnetic resonance imaging. Neuro Oncol 17:1307-1308. doi: 10.1093/neuonc/nov120[doi]

10. Stensjoen AL, Solheim O, Kvistad KA, Haberg AK, Salvesen O, Berntsen EM (2015) Growth dynamics of untreated glioblastomas in vivo. Neuro Oncol 17:1402-1411. doi: 10.1093/neuonc/nov029[doi]

11. Wang CH, Rockhill JK, Mrugala M, Peacock DL, Lai A, Jusenius K, Wardlaw JM, Cloughesy T, Spence AM, Rockne R et al (2009) Prognostic significance of growth kinetics in newly diagnosed glioblastomas revealed by combining serial imaging with a novel biomathematical model. Cancer Res 69:9133-9140. doi: 10.1158/0008-5472.CAN-08-3863[doi]

12. Fan Z, Liu Y, Li S, Liu X, Jiang T, Wang Y, Wang L (2020) Association of tumor growth rates with molecular biomarker status: a longitudinal study of high-grade glioma. Aging 12:7908-7926. doi: 10.18632/aging.103110[doi]

13. Nakasu S, Nakasu Y, Fukami T, Jito J, Nozaki K (2011) Growth curve analysis of asymptomatic and symptomatic meningiomas. J Neurooncol 102:303-310. doi: 10.1007/s11060-010-0319-1[doi]

14. Lee EJ, Kim JH, Park ES, Kim YH, Lee JK, Hong SH, Cho YH, Kim CJ (2017) A novel weighted scoring system for estimating the risk of rapid growth in untreated intracranial meningiomas. J Neurosurg 127:971-980. doi: 10.3171/2016.9.JNS161669[doi]

15. Nakasu S, Fukami T, Nakajima M, Watanabe K, Ichikawa M, Matsuda M (2005) Growth pattern changes of meningiomas: long-term analysis. Neurosurgery 56:946-955 discussion 946

16. Louis DN, Perry A, Wesseling P, Brat DJ, Cree IA, Figarella-Branger D, Hawkins C, Ng HK, Pfister SM, Reifenberger $\mathrm{G}$ et al (2021) The 2021 WHO Classification of Tumors of the Central Nervous System: a summary. Neuro Oncol 23:1231-1251. doi: 10.1093/neuonc/noab106[doi]

\section{Tables}

Table 1,2 is available in the Supplemental Files section.

\section{Figures}



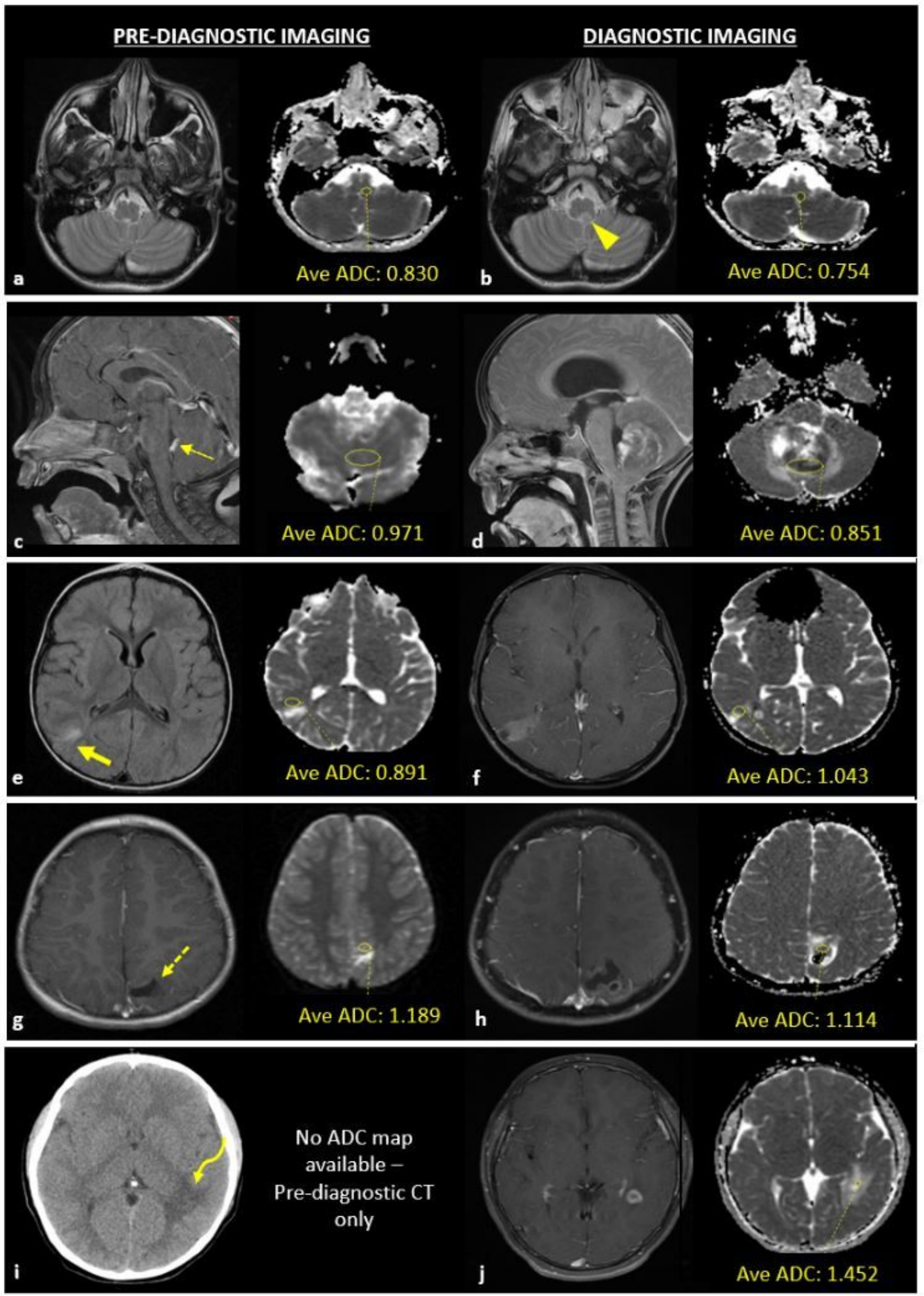

Figure 1

Example of apparent diffusion coefficient (ADC) acquisition for a patient with a case of central nervous system lymphoma (a)-(b) and examples of neuroimaging studies with abnormal findings at the site of pediatric brain tumor development (c)-(j). (a) $\mathrm{T}_{2}-\mathrm{W}$ MRI sequence of pre-diagnostic neuroimaging without a macroscopic tumor present and corresponding ADC map with a yellow circle that delineates the area used to calculate average ADC value of the tissue region in which a brain tumor would later develop; (b) 
$\mathrm{T}_{2}$-W MRI sequence obtained at the time of diagnosis demonstrating the macroscopic lesion (arrowhead) and corresponding ADC map with a yellow circle that delineates the area of the tumor used to calculate average ADC value. (c) Pre-diagnostic contrast enhanced (CE) - $T_{1}$ WI sagittal image showing thickened, enhancing choroid (thin arrow) and pre-diagnostic ADC map; (d) post-diagnostic CE- $\mathrm{T}_{1}$ WI sagittal image and ADC map of atypical teratoid rhabdoid tumor (ATRT). (e) Pre-diagnostic $T_{2}$ /FLAIR-WI axial image showing hyperintense $T_{2}$ /FLAIR signal (thick arrow) and pre-diagnostic ADC map; (f) post-diagnostic CE $\mathrm{T}_{1} \mathrm{WI}$ axial image with $\mathrm{ADC}$ map of low-grade glioma. (g) Pre-diagnostic $C E-\mathrm{T}_{1} \mathrm{WI}$ axial image showing encephalomalacia/gliosis (dashed arrow) and pre-diagnostic ADC map; (h) post-diagnostic CE - $\mathrm{T}_{1} \mathrm{WI}$ axial image with ADC map of a ganglioglioma. (i) Pre-diagnostic axial CT image showing white matter hypoattenuation (curved arrow); no pre-diagnostic ADC map was available due to lack of MR imaging; (j) post-diagnostic CE-T 1 WI axial image with ADC map of JXG. 

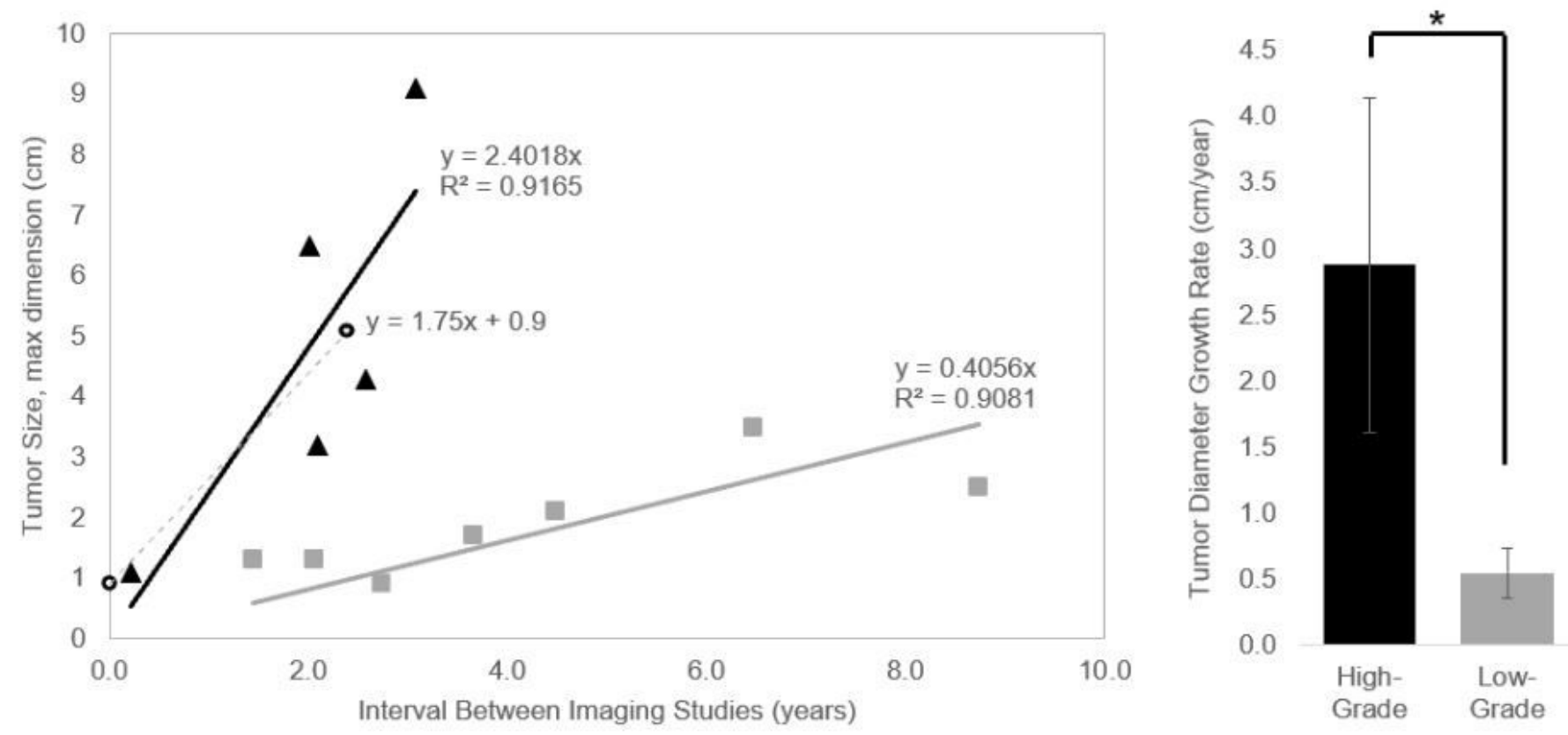

a.

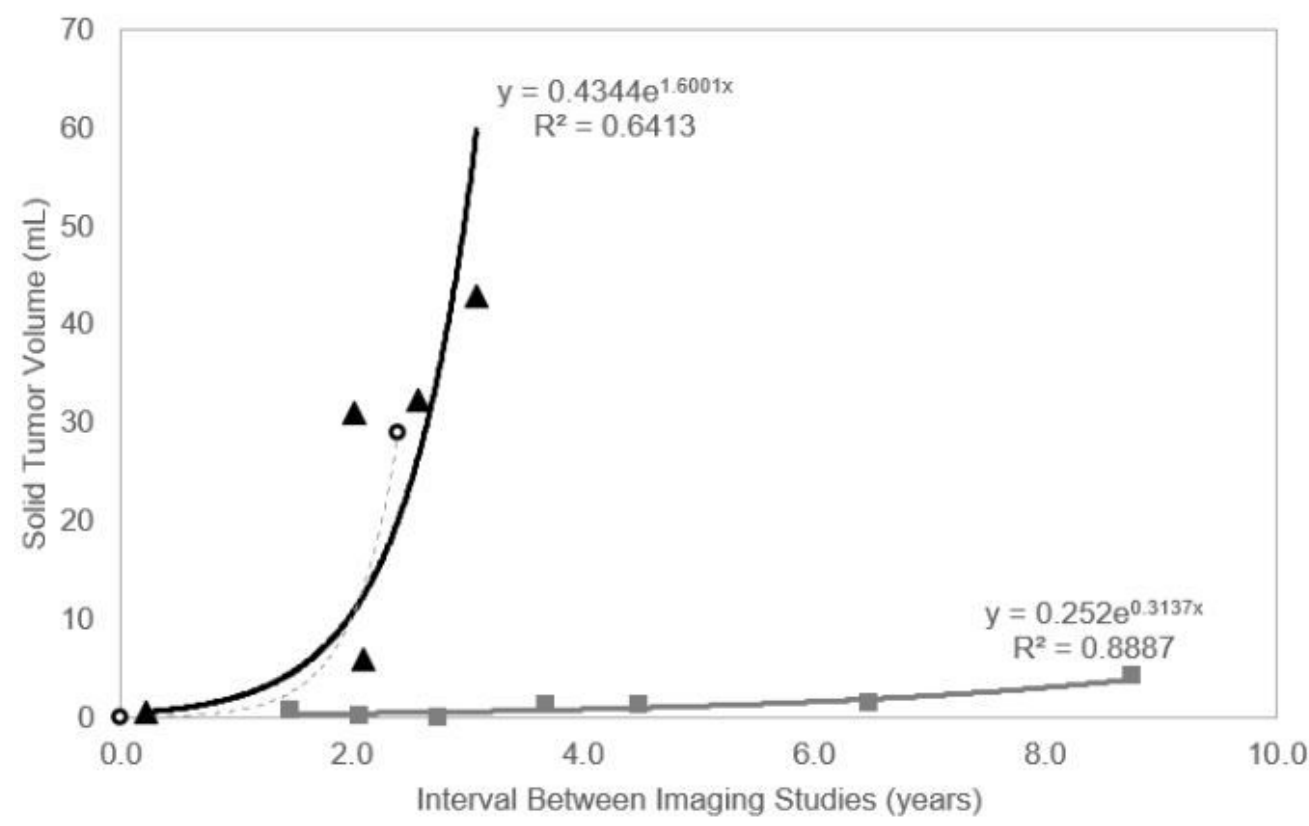

c. b.

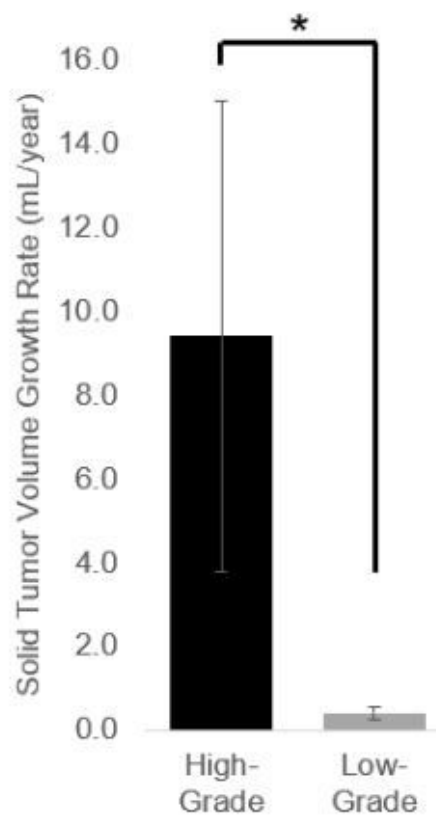

d.

* $p$-value $\leq 0.05$ showed statistical significance.

\section{Figure 2}

(a) Linear regression model for growth of the measured diameter length. Tumor diameter as a linear function of the time interval between pre-diagnostic and diagnostic imaging for high-grade tumors (triangle), low-grade tumors (square), and ATRT reference (circle). (b) Tumor diameter growth rate of the high-vs low-grade tumors is significantly different $(p=0.02)$. (c) Exponential regression model for growth of the measured solid tumor volume (i.e., constant volume doubling time). Calculated solid tumor volume 
as an exponential function of the time interval between pre-diagnostic and diagnostic imaging for highgrade tumors (triangle), low-grade tumors (square), and ATRT reference (circle). (d) Solid tumor volume growth rate of the high- vs low-grade tumors is significantly different $(p=0.03)$

n.s.

1.8

1.6

1.4

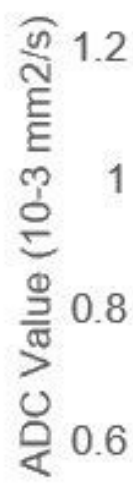

0.4

0.2

0

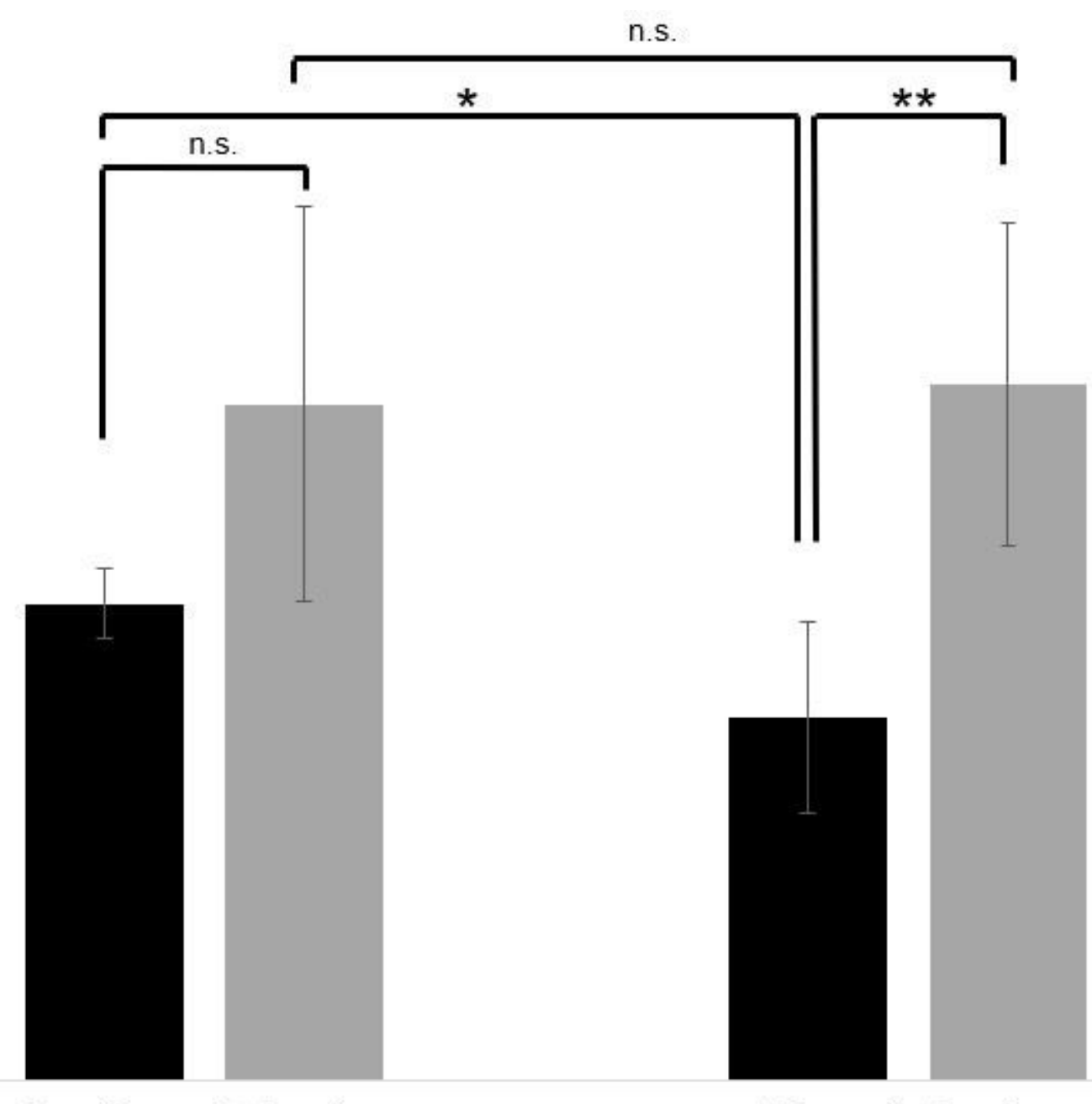

Pre-diagnostic Imaging

Diagnostic Imaging

- High-Grade = Low-Grade

\footnotetext{
* Significant, $p$-value $\leq 0.05$

*** Very significant, $p$-value $\leq 0.01$

n.s. $=$ not significant
}

\section{Figure 3}

Apparent diffusion coefficient (ADC) values in the corresponding pre-diagnostic regions of tumor growth compared to $A D C$ values of the tumor at the time of diagnosis. $A D C$ values of high-grade tumors were lower on diagnostic imaging than the corresponding region on pre-diagnostic imaging $(p=0.05)$. For lowgrade tumors, $A D C$ values for low-grade tumors were not significantly different between pre-diagnostic and diagnostic imaging $(p=0.87)$. On pre-diagnostic imaging, there was no significant difference between ADC values of high- and low-grade tumors $(p=0.22)$. However, at time of diagnosis, the ADC values for the high-grade tumors was lower than the low-grade tumors $(p=0.002)$. 


\section{Supplementary Files}

This is a list of supplementary files associated with this preprint. Click to download.

- Table1.jpg

- Table2.jpg 